\title{
Using Three-Dimensional Gait Data for Foot/Ankle Orthopaedic Surgery
}

\author{
Gwyneth de Vries ${ }^{1}$, Kevin Roy $^{2}$ and Victoria Chester ${ }^{*}, 2$ \\ ${ }^{I}$ Department of Orthopaedic Surgery, Dr. Everett Chalmers Hospital, Fredericton, New Brunswick, Canada \\ ${ }^{2}$ Faculty of Kinesiology, University of New Brunswick, Fredericton, New Brunswick, Canada
}

\begin{abstract}
We present the case of a forty year old male who sustained a torn carotid during strenuous physical activity. This was followed by a right hemispheric stroke due to a clot associated with the carotid. Upon recovery, the patient's gait was characterized as hemiparetic with a stiff-knee pattern, a fixed flexion deformity of the toe flexors, and a hindfoot varus. Based on clinical exams and radiographs, the surgical treatment plan was established and consisted of correction of the forefoot deformities, possible hamstrings lengthening, and tendon transfer of the posterior tibial tendon to the dorsolateral foot. To aid in surgical planning, a three-dimensional gait analysis was conducted using a state-of-the-art motion capture system. Data from this analysis provided insight into the pathomechanics of the patient's gait pattern. A forefoot driven hindfoot varus was evident from the presurgical data and the tendon transfer procedure was deemed unnecessary. A computer was used in the OR to provide surgeons with animations of the patient's gait and graphical results as needed. A second gait analysis was conducted 6 weeks post surgery, shortly after cast removal. Post-surgical gait data showed improved foot segment orientation and position. Motion capture data provides clinicians with detailed information on the multisegment kinematics of foot motion during gait, before and during surgery. Further, treatment effectiveness can be evaluated by repeating gait analyses after recovery.
\end{abstract}

Keywords: Motion capture, gait, multisegment foot model, kinematics.

\section{INTRODUCTION}

Clinical gait analysis typically uses state-of-the-art motion capture systems to provide information on pathological walking patterns. Gait analysis has been used extensively in the surgical treatment planning of children with Cerebral Palsy (CP). Based on this population, various outcome studies have shown that gait analyses often change the surgical plan for patients [1-4]. Given the success of gait data in pediatric CP populations, we questioned whether clinical gait analysis would be beneficial for foot/ankle surgical treatment planning in adults.

Movements of the foot are difficult to measure, mainly because of the complexity and small size of the segment. As a result, most biomechanical studies have modeled the foot as a single rigid segment. Recently, improved motion capture systems have led to an increase in the number of studies that model the foot as a multisegment system [5-8]. For camerabased motion capture systems, such models present numerous challenges. Reflective markers are used to track various anatomical landmarks on the foot during walking. The close proximity of these markers can cause problems with marker identification, merging, and crossover. For these reasons, the use of motion capture to further understand multisegment foot kinematics is still a relatively new endeavor.

Additional challenges associated with the use of motion capture systems to track foot motion include the movement of skin relative to the underlying bone, differential

*Address correspondence to this author at the Faculty of Kinesiology, University of New Brunswick, Peter Kelly Drive, Fredericton, New Brunswick, Canada; E-mail: vchester@unb.ca movement of the bones across foot segments (failure of the rigid body assumption), and marker set configuration, all of which contribute to the overall error. Nester et al. [9] examined these issues by comparing the kinematic data provided by: 1) bone anchored markers, 2) markers attached to rigid plates, which were mounted on the skin surface, and 3) markers attached directly to the skin for four foot segments (heel, navicular/cuboid, medial and lateral forefoot). The study found that there were no systematic differences between skin and plate marker protocols and bone motion. Therefore, it was suggested that no one particular rigid body foot model or marker attachment protocol is preferred. This work suggests that the measurement of multisegment foot motion is feasible using motion capture systems.

Clinical gait analysis facilitates the investigation of foot motion during a dynamic weightbearing task. As such, gait analysis may provide a better measure of foot functionality than traditional clinical measures, providing additional insight for treatment planning. Therefore, using a case study analysis, our goal was to examine whether multisegment foot kinematic data aids in surgical planning and treatment evaluation for foot/ankle disorders.

\section{MATERIALS AND METHODOLOGY}

A thirty-five year old male patient sustained a right carotid artery dissection during strenuous physical activity. Prior to this, the patient was otherwise in good health. The patient suffered left hemiparesis as a result of the injury and demonstrated a hemiparetic gait. Following initial rehabilitation efforts, the patient had an equinovarus position of the foot that was addressed by Achilles tendon lengthening and split tibialis anterior tendon transfer. With 
the foot restored to a plantigrade position, the patient was able to pursue more aggressive gait rehabilitation. Rehabilitation reached a plateau when excessive flexion through the hallux interphalangeal joint and interphalangeal joints of the lesser toes became problematic. The clinical impression during heel strike and stance was that of the hallux deformity "posting" the forefoot in varus, and apparent ankle inversion and loss of balance. The clinical impression also indicated some degree of hamstrings contracture and possible flexion contracture at the hip joint. Surgical planning based on the clinical findings included correction of the forefoot deformities, transfer of the posterior tibial tendon to the dorsolateral foot, and possible hamstrings lengthening.

At the age of forty years old (height $=68$ ", weight $=76.8$ $\mathrm{kg}$ ), the patient was referred to the Motion Lab at the University of New Brunswick for a pre-surgical gait analysis. We used an eight-camera Vicon MCam system (Oxford Metrics Group Inc., USA) to capture the threedimensional trajectories of 17 reflective markers placed on the foot and tibia (Fig. 1). Motion data was sampled at a frequency of $120 \mathrm{~Hz}$. The foot was assumed to consist of the following rigid body segments: a) the shank (including the tibia and fibula), b) the rearfoot (including the calcaneus and talus), c) the forefoot (including the metatarsus and phalanges), d) the midfoot (including the navicular, cuboid, and cuneiforms), and e) the foot (including the rearfoot, midfoot, and forefoot). With the exception of the calcaneus, each foot segment could be reliably defined through palpation of key anatomical landmarks. Segment definitions and marker locations are provided in Table $\mathbf{1 .}$
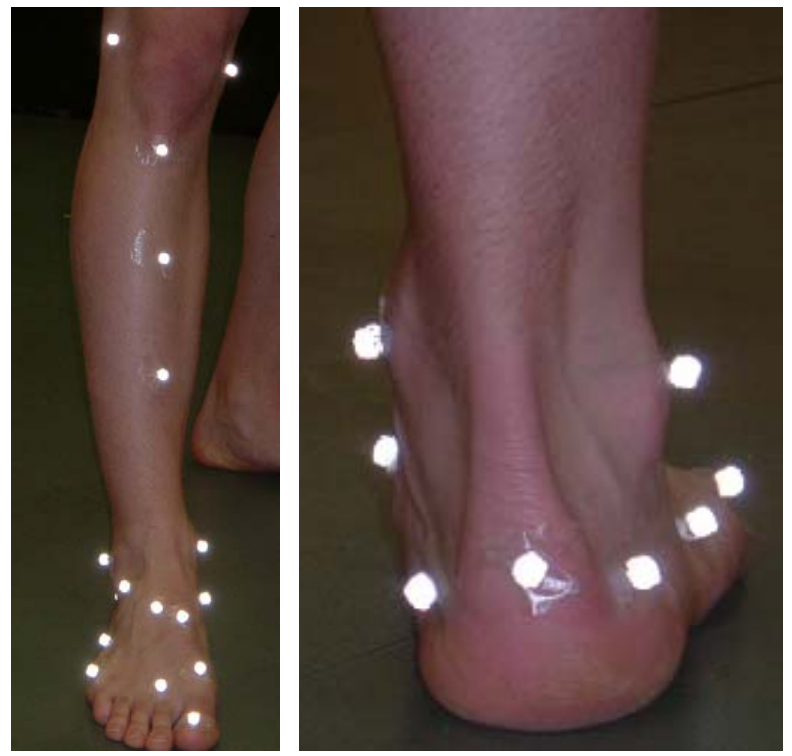

Fig. (1). Front and rear view photos showing locations of foot markers.

To provide a reliable definition of the calcaneus, we created a level plane using the posterior, medial, and lateral calcaneus markers. While the average calcaneal pitch angle is 20 degrees, this angle is difficult to determine without radiographs. Radiographs were not used as they provide no information on the change in this angle with dynamic motion. Instead, we devised a method to create a level
Table 1. Segment Definitions and Marker Locations

\begin{tabular}{|c|l|}
\hline Segment & \multicolumn{1}{|c|}{ Marker Locations } \\
\hline \hline Tibia & $\begin{array}{l}\text { Tibial tuberosity, medial and lateral tibial condyles, anterior } \\
\text { tibia }\end{array}$ \\
\hline Foot & $\begin{array}{l}\text { Medial and lateral malleoli, posterior calcaneus,1st and 5th } \\
\text { distal metarsals }\end{array}$ \\
\hline Rearfoot & Posterior, medial and lateral calcaneus \\
\hline Midfoot & Navicular, cuboid, 1st and 5th proximal metatarsals \\
\hline Forefoot & Hallux, 1st and 5th proximal and distal metatarsals \\
\hline
\end{tabular}

calcaneal plane. While standing on one limb, the height of the lateral malleolus of the supporting limb, with respect to the floor, was measured using calipers. Fifty percent of this height was used to establish the placement of the lateral calcaneus marker underneath the lateral malleolus. Using this lateral calcaneus marker as a guide, crosshair lasers were used to establish the locations of the posterior and medial calcaneus markers (Fig. 2). Using the 3D marker locations, embedded coordinate systems were creating at the knee joint, ankle joint, and at each foot segment. Knee and ankle joint centers were defined as the midpoint between the tibial condyles and malleoli, respectively. Embedded coordinate systems were similar to those outlined by Leardini et al. with the exception of the midfoot segment. Leardini defined the midfoot using: 1) the navicular tuberosity, 2) the middle cuneiform (assumed to coincide with the base of the second metatarsal), and 3) the cuboid tuberosity (assumed to coincide with the base of the fifth metatarsal). Our midfoot segment model differed slightly in terms of the markers used to define the segment. Mainly, we did not assume that the marker location for the cuboid coincided with the base of the fifth metatarsal head. These two anatomical locations were regarded as separate in our definition of the midfoot. The anatomical reference frame or embedded coordinate system for the midfoot segment was then defined as follows:

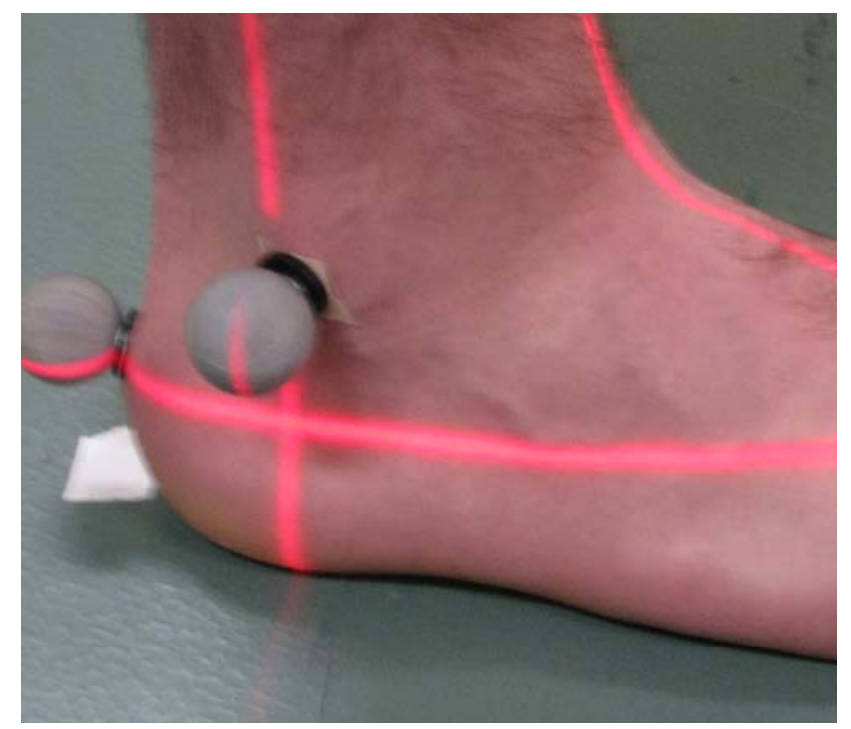

Fig. (2). Laser technique for leveling of calcaneal plane. Cross hairs mark the location of the lateral calcaneus marker. 
Origin Midpoint between the navicular and cuboid

$\mathrm{x}$-axis Vector from the origin to base of the second metatarsal (medial aspect of the second metatarsocuneiform joint);

y-axis Lies in the transverse plane containing the markers for the navicular, cuboid, and base of the second metatarsal;

z-axis Orthogonal to the xy plane.

All embedded coordinate systems were designed in a similar manner using the respective markers for each segment. Joint angles were computed from the relative orientations of the embedded coordinate systems using Euler angles in a yxz sequence, corresponding to flexion/extension, adduction/abduction, and internal/external rotation. Displacement data were filtered using a $6 \mathrm{~Hz}$ low-pass Butterworth filter. All data were normalized to $100 \%$ of the gait cycle. Temporal-spatial measures were computed for each gait cycle using the Vicon motion capture data. Cadence, velocity, and percent of cycle spent in single stance were calculated for each gait cycle. The single gait cycle that most closely approximated the individual mean of all gait cycles on these three measures was selected as the single trial for analysis. Patient data was compared to a single control subject (male; age $=30$ years; height $=69$ "; weight $=84.1 \mathrm{~kg})$.

During the surgical procedure, a laptop computer was used for viewing of the patient's gait data and multimedia files. Multimedia files included video images of the patient walking (sagittal and frontal views), as well as 3D animated skeletal images of the patient (Fig. 3). Accessibility to this information during surgery helps minimize the clinician's reliance on memory of the patient's movements.

Six weeks post-surgery, the patient was invited to participate in a second gait analysis. While ankle casts had only been removed a few days prior, this was the first of a series of post-surgical assessments. Six week post-surgery data is provided in this study.

\section{RESULTS}

\section{Clinical Examinations}

\section{Physical Assessment (Findings Awake)}

The patient's affected foot was free of edema and had normal pulses and preserved sensation. Previous surgical scars at the Achilles tendon, medial arch, and dorsolateral foot were well healed. Range of motion at the ankle showed dorsiflexion of approximately 10 degrees and plantar flexion of approximately 30 degrees. Manipulation of the subtalar joint showed preserved motion through the joint comparable to the unaffected side. The midfoot was mobile with respect to the hindfoot, again comparable to the unaffected side. The previous split anterior tendon procedure was appreciated by direct palpation revealing the lateral aspect of the tendon to be firmly anchored at the dorsolateral foot with appropriate tissue tension, thus contributing to the perceived normal motion of the midfoot with respect to the hindfoot.

Active motion of the foot showed strong active dorsiflexion using the split tibialis anterior augmented by activation of extensor hallux longus and extensor digitorum longus tendons. Inversion of the foot revealed an intact and powerful tibialis posterior tendon. The forefoot showed physiologic motion at the tarsal-metatarsal junction, again comparable to the unaffected side. Findings at the metatarsal-phalangeal joints and toes were abnormal with flexion deformities that were only partially correctable through passive manipulation. In particular, there was flexion of the hallux at the interphalangeal joint and flexion of the lesser toes at the proximal and distal interphalangeal joints. Range of motion of the hip joint was normal with no hip flexion contracture. Range of motion of the knee was found to be restricted due to increased muscle tone. Even with full cooperation of the patient, it was difficult to fully flex or fully extend the knee due to muscular co-contraction of the quadriceps and hamstring groups.

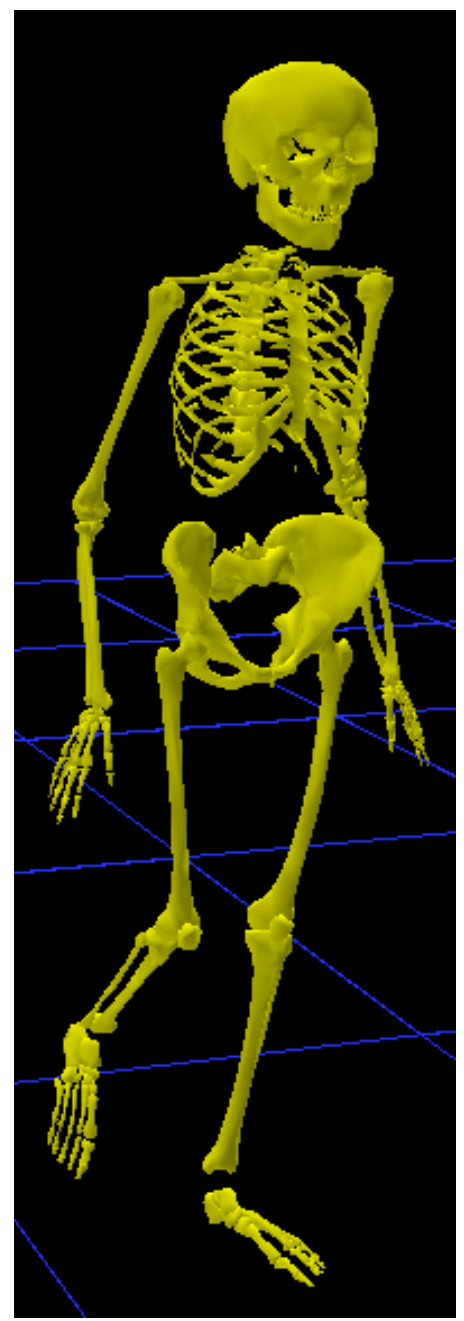

Fig. (3). Image of the animated skeleton of the patient.

Weightbearing showed a neutral position of the unaffected foot with neither flatfoot nor cavovarus foot position. On the affected side, weightbearing was accomplished through the heel, the lateral midfoot, and the forefoot with the important finding of flexion of the hallux at the interphalangeal joint. The lesser toes did contact the ground normally, but in a slightly flexed posture with increased ground contact of the toe tips. The position of the hallux thus tilted the forefoot into slight supination. The midfoot also appeared to have slight supination and the heel was noted to be in varus (Fig. 4). Performing the Coleman block test 
(placing a $1 \mathrm{~cm}$ block under the heel and lateral border of the foot) showed that the heel could be reduced to a normal (i.e. slightly valgus position).
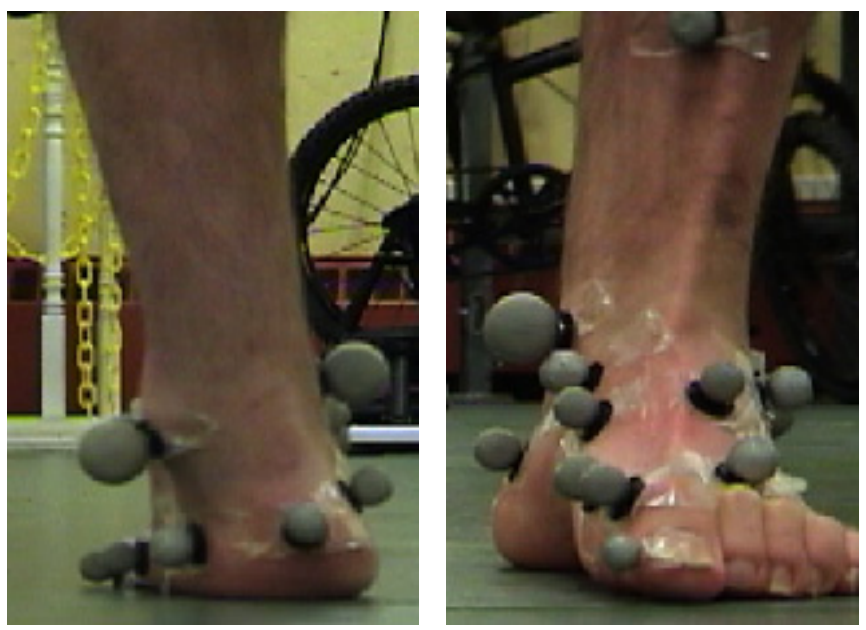

Fig. (4). Rear and front view of the patient's foot, demonstrating a hindfoot varus.

\section{Radiographs}

Standard antero-posterior, oblique and lateral views of the foot, plus anteroposterior and lateral views of the ankle showed the foot and ankle to be free from any areas of joint space narrowing or degenerative features. Specifically, there was no subtalar, midfoot, metatarsal-phlalangeal nor interphalangeal joint arthritis/anklylosis to account for the clinical findings.

Based on the results of the physical examination and radiographs, the patient was thought to have several problems contributing to his gait abnormalities. The first was possible joint contracture at the knee, requiring examination under anaesthesia and possible hamstrings lengthening. The second was forefoot deformity of excessive toe flexion to all toes (the hallux interphalangeal joint in particular). The final finding was hindfoot and midfoot varus, either due to toe deformity alone or due to the toe deformity plus excess tone of the foot invertors (tibialis posterior, flexor digitorum longus). To address the forefoot flexion deformities, the flexor hallux longus tendon would be released from the distal phalanx of the great toe and sutured to the base of the proximal phalanx. The flexor digitorum longus tendons to the lesser toes would be released using plantar toe incisions. Transfer of the posterior tibialis tendon to the lateral portion of the midfoot was planned to reduce the hindfoot varus deformity and provide dorsiflexion assistance.

\section{Pre-Surgical Gait Analysis}

The patient presented with a hemiplegic (left side) gait pattern. A stiff knee gait pattern and flexion deformities at the toe contributed to difficulty achieving sufficient foot clearance during the left limb swing phase. Despite hip hiking and circumduction to aid in foot clearance, dragging of the hallux at toe-off was observed. With respect to the tibia, the foot segment demonstrated a mainly dorsiflexed, externally rotated, and supinated orientation throughout the gait cycle. Weightbearing occurred mainly on the lateral border of the foot and the toe tips.
Patient multisegment foot kinematic data during a gait cycle was compared to a control subject. In the control subject, the calcaneus was everted with respect to the tibia for the majority of stance phase until terminal stance, at which point, the calcaneus was inverted (Fig. 5). In contrast, the pre-operative data for the clinical patient showed an everted calcaneus at initial contact, followed immediately by inversion of the calcaneus for the remainder of the stance phase. To determine if the calcaneal inversion was due to the toe deformities alone or due to the toe deformities plus excess tone of the foot invertors, we examined the motion of the midfoot with respect to the calcaneus and ankle dorsiflexion angles. Dynamically, the patient's midfoot was everted with respect to the calcaneus for the majority of the stance phase of the gait cycle (Fig. 6). The control subject also showed midfoot eversion during the stance phase but to a lesser extent. The low range of motion and consistent pattern of motion of the midfoot with respect to the calcaneus indicated that throughout the stance phase, the midfoot maintained a relatively fixed position. No indications of excess tone of the invertors were evident.

Due to the hindfoot varus and foot supination, the ankle dorsiflexion angles are not reliable. However, slow motion video analysis confirmed that dorsiflexion was maintained for most of the gait cycle. These results suggest ample dorsiflexion ability. Prior to the gait analysis, the amount of dorsiflexion achieved during gait was of concern. It is likely that the dragging of the hallux on the ground during push-off gave the impression of insufficient dorsiflexion.

Typically, hindfoot varus (calcaneal inversion) would lead to forefoot pronation to maintain contact with the ground. Due to the toe deformities, the patient is unable to bring the medial side of the foot in contact with the ground during part of the stance phase. Therefore, with respect to the calcaneus, the forefoot maintained a supinated position until opposite footstrike (Fig. 7). Once opposite footstrike occurred, and weightbearing was shared across the two limbs, the forefoot began to pronate in preparation for toeoff. In the control subject, the forefoot was supinated with respect to the calcaneus for the majority of stance phase until terminal stance, at which point, the forefoot was pronated. It is likely that pronation occurred earlier in the patient to alter the unstable foot position.

\section{Surgery}

Prior to surgery, radiographs, gait data, and motion capture images were reviewed and interpreted. Based on the kinematic data provided from the gait analysis, the surgical plan was modified. Under anaesthesia, the patient was found to normal knee range of motion, entirely without flexion contracture. No hamstrings tendon lengthening was required. Additionally, the hip was found to have normal range of motion and the absence of contracture was confirmed. The range of motion of the ankle, hindfoot, and midfoot was confirmed to match the preoperative clinical assessment. There was no suggestion of excess midfoot motion that might have been masked by muscle tension in the nonanaesthetized state. An intraoperative review of the gait findings (midfoot compared to hindfoot) was performed to carefully rule out any portion of the gait cycle where 


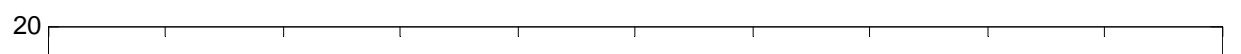

$15-$

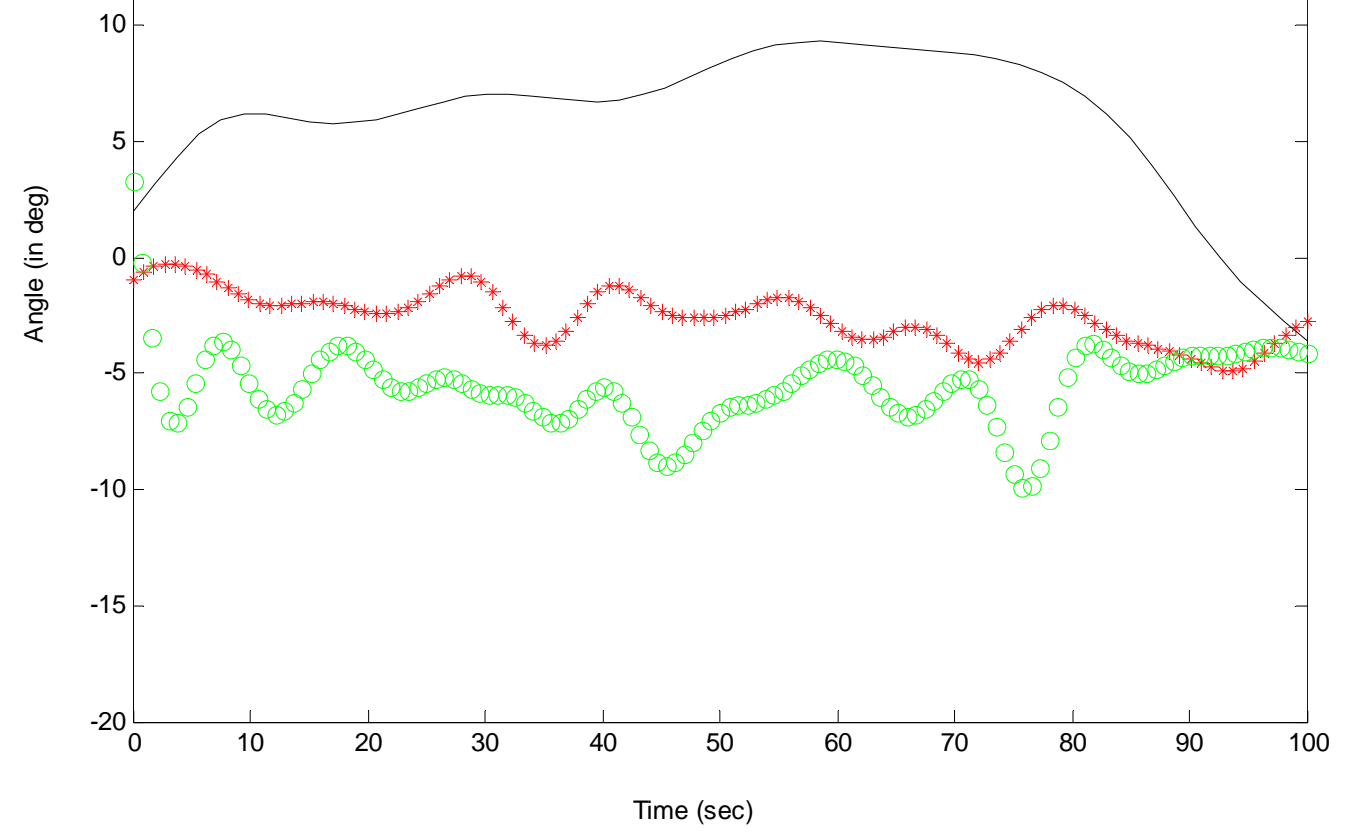

Fig. (5). Calcaneal eversion(+)/inversion(-) with respect to the tibia during the stance phase of gait. Preoperative data (green circles); postoperative data (red stars); normative subject (solid line).

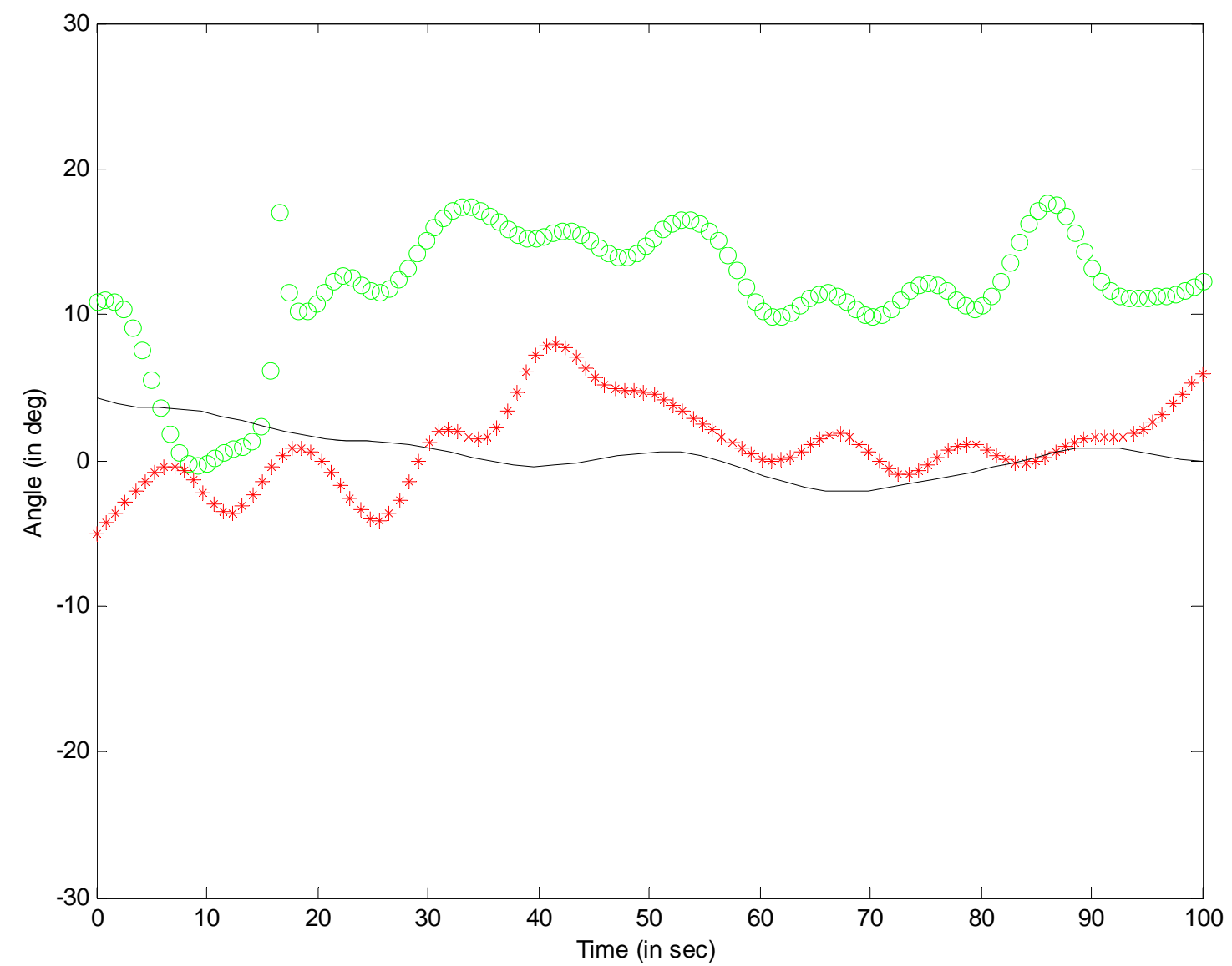

Fig. (6). Midfoot eversion(+)/inversion(-) with respect to the calcaneus during the stance phase of gait. Preoperative data (green circles); postoperative data (red stars); normative subject (solid line). 
increased tibialis posterior activity might be present. Initially, a transfer of the posterior tibialis tendon to the lateral portion of the midfoot was planned to reduce the hindfoot varus deformity and provide dorsiflexion assistance, however, the review of both midfoot motion and ankle dorsiflexion performance confirmed that transferring the tibialis posterior tendon would not be necessary. The forefoot tendon procedures were then performed, removing the deformity at the toes.

A simulated weight bearing position of the foot was then achieved by placing the foot against a flat surface. The desired slight valgus position of the heel was achieved with the midfoot and metatarsal heads also in desired position. The toes contacted the flat surface with the plantar pulp of each toe as anticipated. We were able to conclude that the preoperative varus hindfoot was due to the forefoot orientation imposed by the tension of the long toe flexors, and the midfoot showed normal relative angles with respect to other compartments of the foot (e.g. forefoot, calcaneus) due to appropriate biomechanics in those areas of the foot.

Preoperative and intraoperative examination of the hip and knee confirmed that full range of motion was possible through these joints.

\section{Post-Surgical Gait Analysis}

Pre-post surgical gait data comparisons showed changes in ankle/foot kinematics as early as 6 weeks. Normalization of the forefoot posture relative to the midfoot and hindfoot facilitated improved ankle range of motion, toe clearance and foot position, leading to improved progression and stability during gait. Post-operatively, the calcaneus maintained a more neutral orientation with respect to the tibia during the stance phase (Fig. 5). Relative to the calcaneus, the midfoot showed a reduced degree of eversion, more closely approximating the control data (Fig. 6). With respect to the calcaneus, the forefoot showed slightly increased inversion post-operatively compared to the control subject (Fig. 7). In addition, the pattern of motion of the forefoot was more consistent with the control subject. Overall, the post-surgical gait data showed improved foot segment position and orientation compared to pre-surgical data.

\section{DISCUSSION}

This work discusses the case of a 40-year old male who presented with a hemiparetic, stiff-knee gait pattern accompanied by a hindfoot varus and deformity of the toe flexors. To determine the underlying causes of the hindfoot varus, a 3D gait analysis using a multisegment biomechanical foot model was conducted prior to surgery. Kinematic data for the hindfoot, midfoot, and forefoot revealed a forefoot driven hindfoot varus due to the fixed flexion deformities. While the midfoot showed an everted orientation with respect to the calcaneus, the pattern of motion of this foot segment suggested that excess tone of the invertors was not present. As a result, the planned transfer of the posterior tibialis tendon to the lateral portion of the midfoot to reduce the hind foot varus deformity was deemed unnecessary.

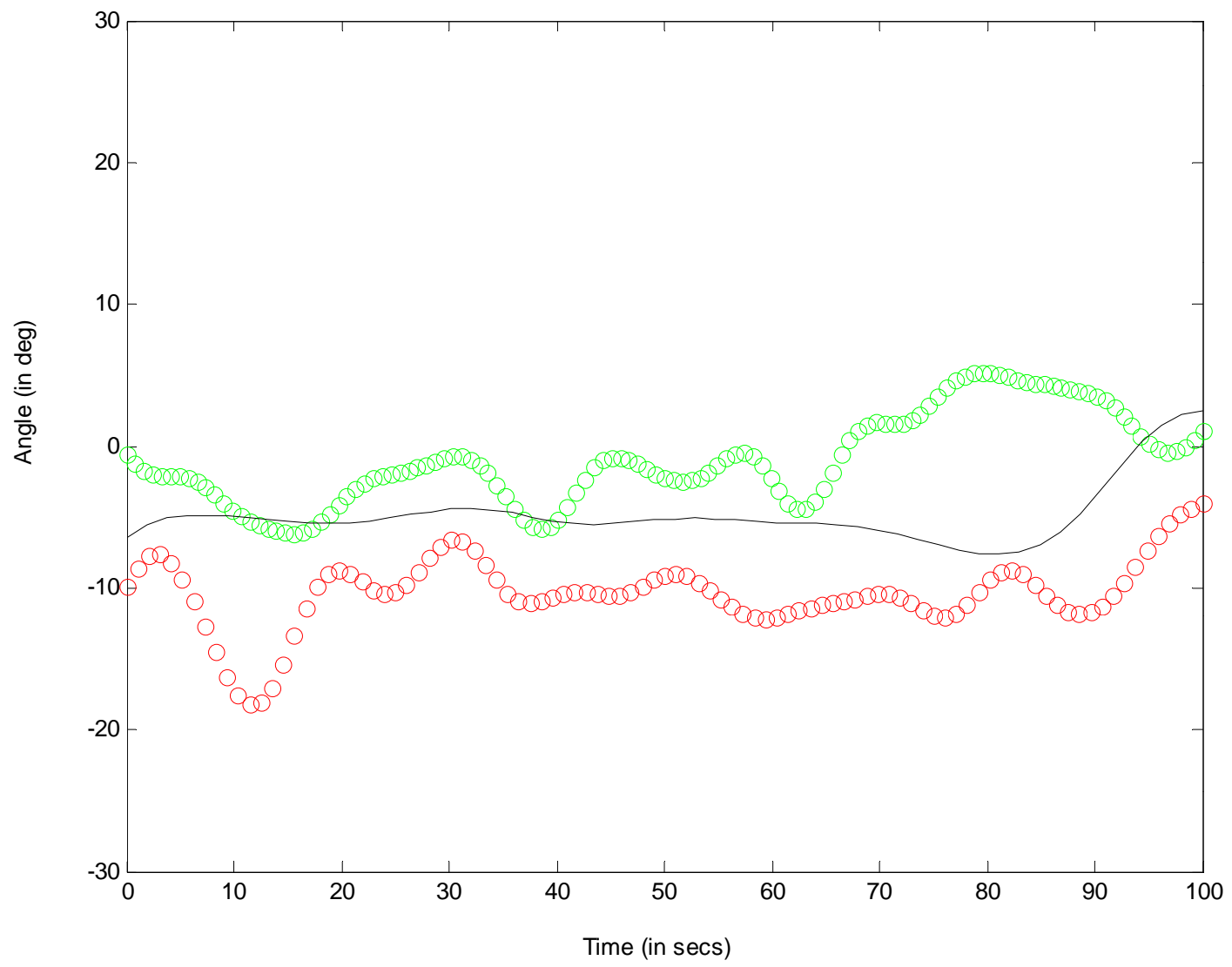

Fig. (7). Forefoot pronation(+)/supination(-) with respect to the calcaneus during the stance phase of gait. Preoperative data (green circles); postoperative data (red stars); normative subject (solid line). 
The origin of hind foot varus can be difficult to isolate using traditional clinical methods. Motion capture studies allow researchers and clinicians to examine the underlying causes of movement disorders (e.g. origin of deformities) and the effectiveness of surgical interventions. Monitoring angles between foot segments during a functional, weight bearing task such as gait provides important information on foot dynamics as a function of time. Unlike traditional clinical methods, gait analysis facilitates the assessment of relative foot motion through a range of angles and weight bearing loads. That is, the behavior of the foot segments can be observed during loading and unloading of the stance limb across a multitude of knee and ankle angles.

\section{CONCLUSIONS}

This work demonstrated the utility of multisegment foot kinematic models for predicting optimal surgical treatments and assessing treatment effectiveness. Continued improvements in motion capture system accuracy and foot biomechanical models will facilitate a greater understanding of normal and pathological foot motion and treatment efficacy.

\section{REFERENCES}

[1] DeLuca PA, Davis RB 3rd, Ounpuu S, et al. Alterations in surgical decision making in patients with cerebral palsy based on threedimensional gait analysis. J Pediatr Orthop 1997; 17(5): 608-14.

[2] Schwartz MH, Viehweger E, Stout J, et al. Comprehensive treatment of ambulatory children with cerebral palsy: an outcome assessment. J Pediatr Orthop 2004; 24(1): 45-53.

[3] Hailey D, Tomie J. An assessment of gait analysis in the rehabilitation of children with walking difficulties. Disabil Rehabil 2000; 22(6): 275-80.

[4] Wren TA, Woolf K, Kay RM. How closely do surgeons follow gait analysis recommendations and why? J Pediatr Orthop B 2005; 14(3): 202-5.

[5] Leardini A, Benedetti MG, Berti L, et al. Rearfoot, md-foot, and fore-foot motion during the stance phase of gait. Gait Posture 2007; 25: 453-62.

[6] MacWilliams BA, Cowley M, Nicholson DE. Foot kinematics and kinetics during adolescent gait. Gait Posture 2003; 17: 214-24.

[7] Carson MC, Harrington ME, Thompson N, O'Connor JJ, Theologis TN. Kinematic analysis of a multi-segment foot model for research and clinical applications: a repeatability analysis. J Biomech 2001; 34: 1299-307.

[8] Woodburn J, Nelson KM, Siegel KL, Kepple TM, Gerber LH. Multisegment foot motion during gait: proof of concept in rheumatoid arthritis. J Rheumatol 2004; 31: 1918-27.

[9] Nester C, Jones RK, Liu A, et al. Foot kinematics during walking using bone and surface mounted markers. J Biomech 2007; 40: $3412-23$

This is an open access article licensed under the terms of the Creative Commons Attribution Non-Commercial License (http://creativecommons.org/licenses/by-nc/ 3.0/) which permits unrestricted, non-commercial use, distribution and reproduction in any medium, provided the work is properly cited. 